\title{
La régulation génétique dans Lactococcus lactis : le modèle de la biosynthèse du tryptophane
}

\author{
A Chopin, J Bardowski, R Raya, SD Ehrlich
}

Laboratoire de génétique microbienne, INRA, 78352 Jouy-en-Josas, France

\begin{abstract}
Résumé - Pour contrôler la fermentation lactique, il est nécessaire de contrôler l'expression des gènes des bactéries lactiques. Pour cela, il faut mieux connaître les modes de régulation génétique de ces bactéries. La régulation de la biosynthèse du tryptophane dans Lactococcus lactis a été étudiée. Les 7 gènes nécessaires à la synthèse du tryptophane ont été clonés et caractérisés. Ils sont présents dans l'ordre trpEGDCFBA, occupent une région de 7968 paires de bases et sont transcrits en un ARN messager unique d'environ $8 \mathrm{~kb}$, indiquant une organisation en opéron. La synthèse de ce messager est modulée d'un facteur 150 par la présence de tryptophane dans le milieu de culture. Le promoteur de transcription a été identifié. De petits ARN messagers sont également produits à partir de ce promoteur. Cependant, leur synthèse n'est pas modulée par le Trp. Le gène lacZ de Escherichia coli qui code pour une $\beta$-galactosidase a été introduit dans le chromosome de $L$ lactis sous le contrôle des signaux d'expression de l'opéron trp. Dans cette souche, la synthèse de $\beta$ galactosidase est modulée par la présence de tryptophane.
\end{abstract}

\section{Lactococcus lactis / tryptophane / biosynthèse / régulation / gène}

Summary - Gene regulation in Lactococcus lactis: the example of tryptophan biosynthesis. To obtain more insight into the mechanisms which control gene expression in Lactococcus lactis, we have undertaken studies on the regulation of the tryptophan (Trp) biosynthetic genes. This model was chosen because the trp genes and their products have been characterized in many prokaryotes and represent a paradigm for the study of gene organization and regulation. The chromosomal trp region from $L$ lactis subsp lactis $L 1403$ was cloned and sequenced. The 7 structural genes are present in the trpEGDCFBA order and span a 7 968-bp segment. Each gene is preceded by a putative ribosome-binding site. Upstream of trpE, a 457-bp non-coding DNA segment contains several regions fitting the consensus for Gram-positive promoters and one region strongly resembling a transcription terminator. Northern hybridisation experiments revealed a 8-kb mRNA transcript, indicating that trp genes are organised in a single operon. The biosynthesis of this transcript was modulated hundred-fold by Trp. Transcription start site was determined by primer extension analysis. Our present data suggest that Trp biosynthesis in L lactis is regulated by modulation of transcription termination. The lacZ gene was introduced into the $\mathrm{L}$ lactis chromosome under the control of the trp expression signals. In this strain, the expression of the $\beta$-galactosidase is modulated by the Trp concentration. 


\section{INTRODUCTION}

Toutes les protéines ne sont pas également abondantes dans la cellule bactérienne. Dans une cellule d'Escherichia coli par exemple, le nombre de molécules de certaines protéines rares est de l'ordre de la dizaine alors que pour des protéines abondantes, ce nombre est de l'ordre de la centaine de milliers (Gouy et Gautier, 1982). Cette différence s'explique principalement par une différence dans la fréquence d'initiation de la transcription des gènes : tandis que certains gènes sont transcrits moins d'une fois par génération, d'autres sont transcrits jusqu'à une fois par seconde (Hoopes et McClure, 1987). En outre, l'expression de la plupart des protéines bactériennes est régulée en fonction des conditions de vie de la cellule. Par exemple, la concentration de $\beta$ galactosidase dans les cellules d'E coli est environ 1000 fois plus élevée en présence qu'en absence de lactose (Beckwith, 1987). Ces phénomènes, qui existent dans toutes les bactéries étudiées, coordonnent l'ensemble de leur activité physiologique et il est important de les connaître pour pouvoir orienter et modifier la physiologie bactérienne.

Cette connaissance serait également importante dans le domaine des bactéries lactiques. II est en effet crucial de pouvoir mieux contrôler les gènes industriels, de façon à obtenir leur expression au niveau optimum, au moment voulu du processus de fabrication. Cependant, les mécanismes qui contrôlent l'expression des gènes des bactéries lactiques n'ont été que peu étudiés, et cela nous a conduits à entreprendre une recherche approfondie sur la régulation génétique dans Lactococcus lactis.

Nous avons choisi comme modèle d'étude le système de biosynthèse du tryptophane (Trp) qui offre plusieurs avan- tages. Les gènes de la voie de biosynthèse du Trp (fig 1), leurs produits et leur régulation ont déjà été caractérisés dans de nombreux organismes (Crawford, 1989) et cela devrait faciliter leur étude dans L lactis. Par ailleurs, le Trp est l'acide aminé dont la biosynthèse est la plus couteuse pour la cellule ( $78 \mathrm{~mol}$ d'ATP sont nécessaires pour la synthèse d'une mole de Trp) (Somerville, 1983). Comme les bactéries lactiques sont énergétiquement limitées, il est probable que l'expression de ces gènes de biosynthèse soit étroitement contrôlée par des mécanismes de régulation efficaces.

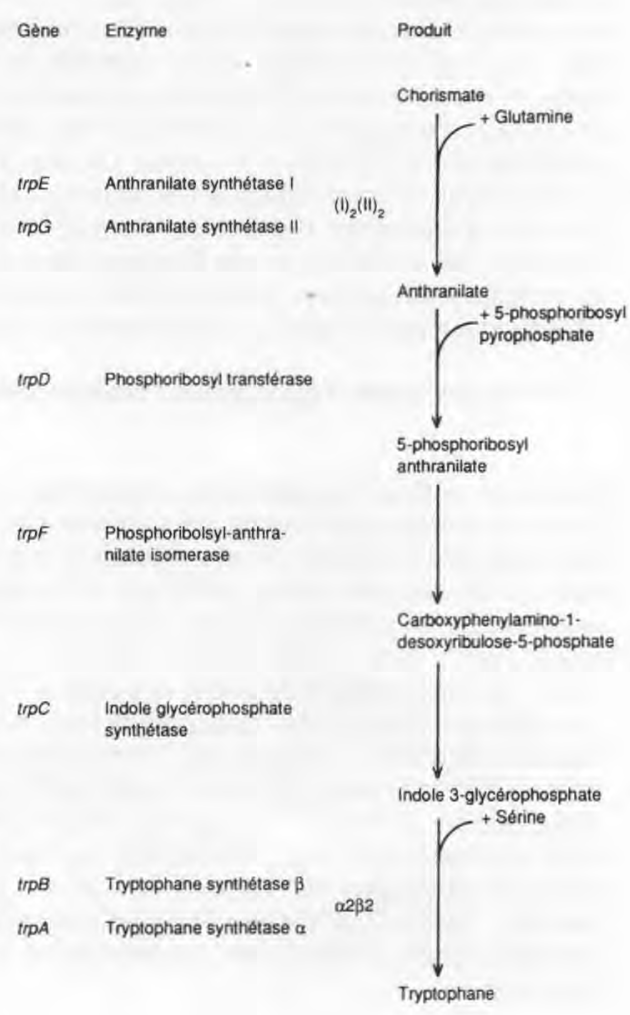

Fig 1. Gènes et enzymes spécifiques de la voie de biosynthèse du tryptophane (d'après Somerville, 1983). 


\section{LES GĖNES DE BIOSYNTHĖSE DU TRYPTOPHANE DE LACTOCOCCUS LACTIS SUBSP LACTIS}

\section{Clonage de la région trp}

La région du chromosome de $L$ lactis subsp lactis contenant les gènes trp, a été clonée dans un mutant Trp- de Bacillus subtilis. Un transformant $\mathrm{Trp}^{+}$a été obtenu dans lequel avait été cloné un fragment d'ADN chromosomique de $L$ lactis subsp lactis IL1403 de 12,7 kilobases (kb) (Bardowski et al, 1992). L'analyse de ce fragment par complémentation de différentes mutations trp de $E$ coli a révélé qu'il complémentait les mutations $\operatorname{trp} A, \operatorname{trp} B, \operatorname{trp} C$ et $\operatorname{trp} D$, mais non $\operatorname{trpE}$. Le clonage de la région adjacente du chromosome de $L$ lactis a permis d'isoler un fragment d'ADN de 3,6 $\mathrm{kb}$, chevauchant partiellement le précédent, et capable de complémenter la mutation trpE de $E$ coli.

\section{Identification des séquences codantes et des enzymes de la voie de biosynthèse}

Les gènes trp et leur organisation ont été caractérisés en détail par séquençage des fragments d'ADN clones. La séquence nucléotidique d'une région de $8,656 \mathrm{~kb}$ a été determinée, dans laquelle 8 séquences codantes probables ont été identifiées (Bardowski et al, 1992). Ces séquences codantes sont précédées, 5 à 7 paires de bases en amont, par des séquences homologues à la séquence de fixation des ribosomes de $L$ lactis (de Vos, 1987). Les séquences codantes potentielles ont été traduites en protéines qui ont été comparées aux protéines de séquences connues. Cela a permis d'établir que 7 de ces 8 séquences codaient pour des protéines ho- mologues aux protéines de biosynthèse du Trp connues chez d'autres organismes, et d'identifier les gènes trp de $L$ lactis subsp lactis. La protéine déduite de la huitième séquence codante potentielle ne présentait d'homologie significative avec aucune séquence protéique connue et a été désignée OrfX. La position des gènes trp de $L$ lactis subsp lactis dans la région étudiée est représentée sur la figure 2.

Les protéines Trp de $L$ lactis subsp lactis présentent de 20 à $61 \%$ d'homologie par rapport aux protéines Trp d'autres organismes (tableau I). La plus faible homologie a été observée dans le cas de TrpF (20 à $34 \%$ ) et la plus forte dans le cas de $\operatorname{TrpB}(50$ à $61 \%)$. L'homologie des protéines Trp n'est pas significativement supérieure entre bactéries phylogénétiquement proches (telles que $L$ lactis subsp lactis et Bacillus subtilis ou Lactobacillus casel) qu'entre organismes phylogénétiquement très éloignés (tels que $L$ lactis subsp lactis et Saccharomyces cerevisiae ou Methanobacterium thermoautotrophicum). Cela est en accord avec le modèle proposé par
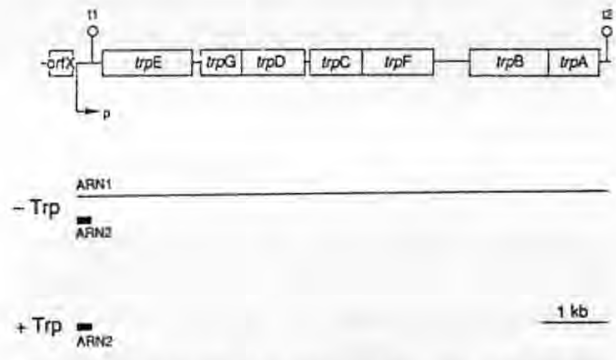

Fig 2. Structure et transcription de la région d'ADN de L lactis subsp lactis IL 1403 contenant les gènes $t r p$. Les gènes sont représentés par des rectangles et les régions d'ADN non codantes sont représentées par une ligne. Dans la partie inférieure sont représentés les ARN messagers transcrits à partir de l'ADN en présence et en absence de tryptophane. L'épaisseur du trait indique une différence dans l'abondance relative des ARN messagers. $p$ : promoteur de transcription; $\mathrm{t} 1$ et $\mathrm{t} 2$ : terminateurs de transcription. 
Tableau I. Homologie de la séquence en acides aminés des enzymes de biosynthèse du tryptophane de Lactococcus lactis subsp lactis avec celles d'autres organismes.

\begin{tabular}{|c|c|c|c|c|c|c|c|c|}
\hline \multirow[t]{2}{*}{ Organismes comparés } & \multicolumn{7}{|c|}{ Identité de séquence (\%) a } & \multirow[t]{2}{*}{ Références } \\
\hline & TrpE & $\operatorname{TrpG}$ & $\operatorname{TrpD}$ & $\operatorname{TrpC}$ & $\operatorname{TrpF}$ & $\operatorname{TrpB}$ & $\operatorname{Trp} A$ & \\
\hline Lactobacillus casei & $-b$ & - & 38 & 38 & 29 & 56 & 32 & Natori et al (1990) \\
\hline Bacillus subtilis & 40 & 44 & 32 & 38 & 24 & 61 & 36 & $\begin{array}{l}\text { Band et al (1984); } \\
\text { Henner et al (1984) }\end{array}$ \\
\hline $\begin{array}{l}\text { Brevibacterium } \\
\text { lactofermentum }\end{array}$ & 33 & 34 & 35 & 32 & 25 & 50 & 25 & Matsui et al (1986) \\
\hline Escherichia coli & 36 & 36 & 35 & 33 & 26 & 54 & 28 & Yanofsky et al (1981) \\
\hline Pseudomonas aeruginosa & 43 & 38 & 36 & 36 & - & 59 & 30 & $\begin{array}{l}\text { Essar et al (1990); } \\
\text { Hadero et Crawford (1986) }\end{array}$ \\
\hline $\begin{array}{l}\text { Methanobacterium } \\
\text { thermoautotrophicum }\end{array}$ & 36 & 40 & 35 & 33 & 20 & 55 & 28 & Meile et al (1991) \\
\hline Saccharomyces cerevisiae & 34 & 36 & 25 & 36 & 34 & 55 & 33 & $\begin{array}{l}\text { Furter et al (1986); } \\
\text { Tschumper et Carbon (1980); } \\
\text { Zalkin et Yanofsky (1982); } \\
\text { Zalkin et al (1984) }\end{array}$ \\
\hline
\end{tabular}

a Cette valeur a été calculée à partir des alignements multiples de séquence obtenus par la méthode de Corpet (1988), en divisant le nombre d'acides aminés conservés à la même position par le nombre d'acides aminés dans la séquence protéique la plus courte; ${ }^{b}$ séquence non publiée.

Crawford (1989) pour l'évolution des protéines $T r p$, selon lequel les régions hautement conservées des protéines sont celles qui sont essentielles pour les fonctions enzymatiques et sont entourées de régions variables dans lesquelles les substitutions d'acides aminés sont plus fréquentes.

\section{Structure et organisation des gènes trp}

Les 7 gènes trp nécessaires à la biosynthèse du Trp sont regroupés sur le chromosome de $L$ lactis subsp lactis. C'est également le cas dans d'autres eubactéries, comme Escherichia coli (Yanofsky et al, 1981), Brevibacterium lactofermentum (Matsui et al, 1986) ou, à un gène près, dans Bacillus subtilis (Henner et al, 1984; Slock et al, 1990) ainsi que dans l'archaébactérie Methanobacterium thermoautotrophicum (Meile et al, 1991). En revanche, dans Pseudomonas, les gènes trp sont répartis dans 4 endroits différents du chromosome (Crawford et Gunsalus, 1966; Calhoun et al, 1973).

Dans $L$ lactis subsp lactis, les 7 gènes trp recouvrent une région de $7,968 \mathrm{~kb}$ et 
sont présents dans l'ordre trpEGDCFBA qui est le plus fréquent chez les bactéries. Tous les gènes trp utilisent AUG comme codon d'initiation de traduction, à l'exception de $\operatorname{trp} G$, qui commence par un codon UUG. $\operatorname{trp} G, \operatorname{trp} F$ et $\operatorname{trp} B$ se terminent par le codon UGA et les 4 autres gènes trp se terminent par le codon UAA. Chaque gène est précédé d'une séquence riche en purines, contenant un site potentiel de fixation au ribosome. Trois paires de gènes, $\operatorname{trp} G$-trpD, $\operatorname{trp} C$-trpF et $\operatorname{trp} B$-trpA, se chevauchent de 11,11 et 4 paires de bases respectivement. Trois autres paires de

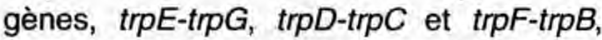
sont séparées par des espaces intercistroniques de 124,46 et 585 paires de bases respectivement. II n'a pas été observé de fusion entre les gènes trp de $L$ lactis subsp lactis.

\section{RÉGULATION DE L'EXPRESSION DES GÈNES $t r p$}

\section{L'expression des gènes trp est régulée par la présence de tryptophane}

L'observation que les gènes trp sont rassemblés dans une région unique du chromosome de $L$ lactis subsp lactis suggère une expression et une régulation coordonnées de ces gènes. Les ARN messagers transcrits à partir des gènes trp ont été analysés dans des expériences d'hybridation par Northern (Raya, en préparation). Les résultats sont schématisés sur la figure 2. En présence de Trp dans le milieu de culture, de petits ARN messagers sont abondamment transcrits. En revanche, en absence de Trp, on observe en plus la transcription d'un ARN messager de $8 \mathrm{~kb}$ environ, représentant la totalité des gènes trp. Ces résultats indiquent que la transcription des gènes trp est contrôlée par la présence de Trp dans le milieu. Le point de départ de la transcription a été déterminé par cartographie à la transcriptase inverse ce qui a permis d'identifier le promoteur de transcription (Raya, en préparation) qui présente les caractéristiques typiques des promoteurs de transcription des lactocoques (de Vos, 1987).

Les points d'arrêts de transcription observés en amont de $\operatorname{trp} E$ et en aval de $\operatorname{trp} A$ coincident avec des séquences caractéristiques des terminateurs de transcription bactériens rho-indépendants (d'Aubenton Carafa et al, 1990). Ces terminateurs de transcription potentiels sont designés $t 1$ et t2 (fig 2).

L'ensemble de ces résultats indique que la biosynthèse du Trp dans $L$ lactis subsp lactis est régulée, au moins en partie, au niveau de la transcription et que les gènes trp qui sont transcrits sous forme d'un ARN messager unique constituent un opéron.

\section{Construction et régulation d'une fusion trpE::lacZ}

Pour mieux caractériser la régulation de l'expression des gènes $t r p$, nous avons mesuré le niveau d'expression de l'opéron trp dans des conditions inductrices et non inductrices. Pour cela, un gène hybride formé de la fusion du début du gène trpE avec le gène lac $Z$ de $E$ coli qui code pour la $\beta$-galactosidase, précédé en amont par la région leader (reg) de l'opéron trp a été intégré dans le chromosome de $L$ lactis subsp lactis, en amont des gènes trp. La méthode utilisée pour cette construction a déjà été décrite (Chopin et al, 1989) et le détail de la construction (Bardowski, en préparation) est donné sur la figure 3.

La production de $\beta$-galactosidase par cette souche a été mesurée dans un milieu de culture synthétique contenant des concentrations croissantes en Trp. Les résultats obtenus montrent que l'expression 

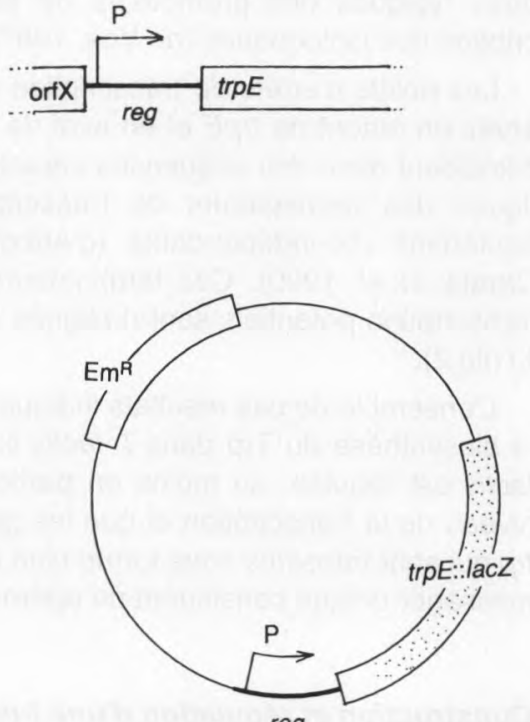

reg

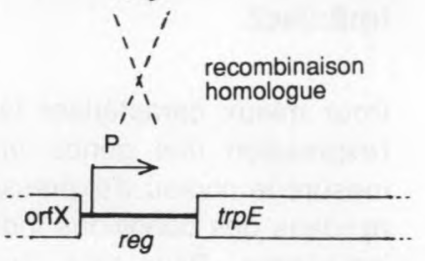

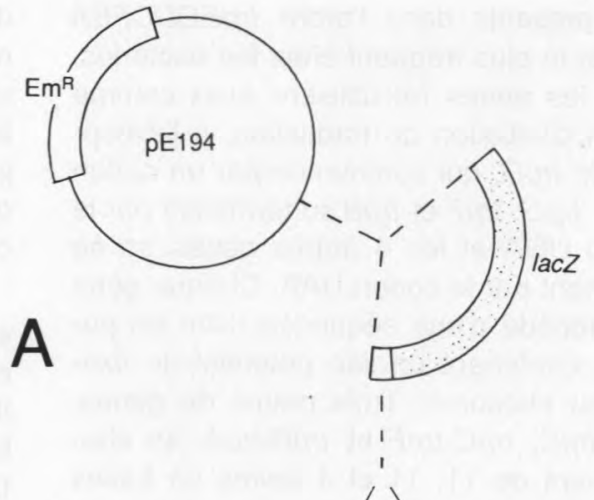
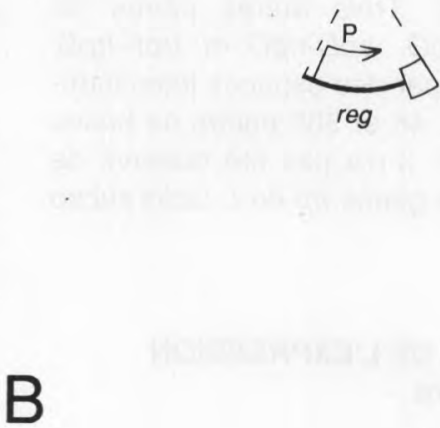

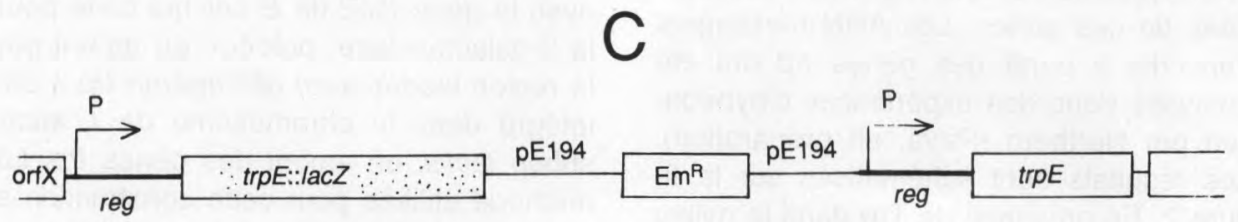

Fig 3. Intégration d'un gène hybride trpE::lac $Z$ dans la chromosome de $L$ lactis subsp lactis IL1403. La méthode utilise la particularité du plasmide pE194 à se répliquer dans Bacillus subtilis, mais non dans Lactococcus lactis (Chopin et al, 1989). A. Un fragment d'ADN contenant la région leader (reg) en amont de trpE et la partie $5^{\prime}$ du gène est cloné en phase en amont du gène lac $Z$ de $E$ coli, puis l'ensemble est inséré dans pE194. Le plasmide résultant est répliqué dans $B$ subtilis, puis utilisé pour transformer $L$ lactis. B. Comme le plasmide est incapable de se répliquer, le seul événement pouvant produire des transformants résistant à l'érythromycine (EmR) est l'intégration du plasmide dans le chromosome par recombinaison homologue. La structure finalement obtenue dans le chromosome est indiquée en $\mathbf{C}$. 
de la fusion est contrôlée par la concentration en Trp. Quand la concentration en Trp est limitante, l'expression du gène hybride (et donc des gènes trp) est augmentée d'un facteur 150. Cela démontre qu'il est possible de contrôler efficacement l'expression d'un gène cloné sur le chromosome de $L$ lactis.

L'ensemble des résultats permet de proposer un modèle expliquant la régulation de la transcription des gènes trp en réponse à la concentration en Trp du milieu. Que le Trp soit présent ou non, une forte transcription est initiée au niveau du promoteur en amont de trpE. En l'absence de Trp, une fraction des transcrits n'est pas arrêtée par le terminateur de transcription $t 1$ et se poursuit à travers les gènes trp jusqu'au terminateur $t 2$ : les gènes trp sont exprimés. En revanche, en présence de Trp, on n'observe plus de transcrits des gènes trp et ceux-ci ne sont plus exprimés. II semble donc que la modulation de la transcription se fasse par la modulation de l'efficacité du terminateur de transcription t1. Ce mécanisme semble différent des mécanismes de régulation déjà caractérisés en détail dans d'autres bactéries et nous poursuivons son étude au niveau moléculaire.

\section{PERSPECTIVES}

Les travaux que nous avons rapportés, ainsi que d'autres actuellement en cours dans notre laboratoire (Godon et al, 1992 ; Delorme et al, 1992; Godon et al, en préparation; Delorme et Renault, en préparation) ont permis d'identifier des mécanismes efficaces de contrôle de l'expression génétique dans $L$ lactis subsp lactis. Dans l'exemple de la biosynthèse du Trp, nous avons montré qu'il était possible d'utiliser l'un de ces mécanismes pour contrôler l'expression d'un gène étranger en réponse à un paramètre du milieu de culture.

L'approche présentée dans cet article, le savoir-faire et les concepts acquis au cours de ce travail pourront prochainement être appliqués à l'étude de la régulation de gènes dont l'expression conditionne l'activité des lactocoques au cours de la transformation fromagère.

\section{REMERCIEMENTS}

Le travail décrit a été financé en partie par le Contrat Bridge-Biot-CT91-0263 du Biotechnology Action Programme de la Commission of the European Communities.

\section{RÉFÉRENCES}

Band L, Shimotsu H, Henner DJ (1984) Nucleotide sequence of the $B$ subtilis trpE and $\operatorname{trp} D$ genes. Gene 27, 55-65

Bardowski J , Erhlich SD, Chopin A (1992) Tryptophan biosynthesis genes in Lactococcus lactis subsp lactis. J Bacteriol 174, 6563-6570

Beckwith J (1987) The lactose operon. In : Escherichia coli and Salmonella typhimurium. Cellular and molecular biology (Neidhardt FC, ed). Am Soc Microbiol, Washington DC

Calhoun DH, Pierson DL, Jensen RA (1973) The regulation of tryptophan biosynthesis in Pseudomonas aeruginosa. Mol Gen Genet 121, 117-132

Chopin MC, Chopin A, Rouault A, Galleron N (1989) Insertion and amplification of foreign genes in the Lactococcus lactis subsp lactis chromosome. Appl Environ Microbiol 55, 1769-17774

Corpet $F$ (1988) Multiple sequence alignment with hierarchical clustering. Nucleic Acids Res 16, 10881-10890

Crawford IP (1989) Evolution of a biosynthetic pathway: the tryptophan paradigm. Annu Rev Microbiol 43, 567-600

Crawford IP, Gunsalus IC (1966) Inducibility of tryptophan synthetase in Pseudomonas putida. Proc Natl Acad Sci USA 56, 717-724 
d'Aubenton Carafa Y, Brody E, Thermes C (1990) Prediction of rho-independent Escherichia coli transcription terminators. A statistical analysis of their RNA stem-loop structures. J Mol Biol 216, 835-858

Delorme C, Ehrlich SD, Renault P (1992) Histidine biosynthesis genes in Lactococcus lactis subsp lactis. J Bacteriol 174, 6571-6579

de Vos WM (1987) Gene cloning and expression in lactic streptococci. FEMS Microbiol Rev 46, 281-295

Essar DW, Eberly L, Han C, Crawford IP (1990) DNA sequences and characterization of four early genes of the tryptophan pathway in Pseudomonas aeruginosa. J Bacteriol 172, 853-866

Furter R, Paravicini G, Aebi M, Braus G, Prantl F, Niederberger P, Hutter R (1986) The TRP4 gene of Saccharomyces cerevisiae: isolation and structural analysis. Nucleic Acids Res 14, 6357-6373

Godon JJ, Chopin MC, Ehrlich SD (1992) Branched chain amino acids genes in Lactococcus lactis subsp lactis. J Bacteriol 174, 6580 6589

Gouy M, Gautier C (1982) Codon usage in bacteria: correlation with gene expressivity. $\mathrm{Nu}$ cleic Acids Res 10, 7055-7074

Hadero A, Crawford IP (1986) Nucleotide sequence of the genes for tryptophan synthase in Pseudomonas aeruginosa. Mol Biol Evol 3, 191-204

Henner DJ, Band L, Shimotsu H (1984) Nucleotide sequence of the Bacillus subtilis tryptophan operon. Gene 34, 169-177

Hoopes BC, McClure WR (1987) Strategies in regulation of transcription initiation. In: Escherichia coli and Salmonella typhimurium. Cellular and molecular biology (Neidhardt FC, ed), Am Soc Microbiol, Washington DC

Matsui K, Sano K, Ohtsubo E (1986) Complete and deduced aminoacid sequences of the
Brevibacterium lactofermentum tryptophan operon. Nucleic Acids Res 14, 10113-10114

Meile L, Stettler R, Banholzer R, Kotik M, Leisinger T (1991) Tryptophan gene cluster of Methanobacterium thermoautotrophicum Marburg: molecular cloning and nucleotide sequence of a putative trpEGCFBAD operon. J Bacteriol 173, 5017-5023

Natori Y, Kanao Y, Imamoto F (1990) Nucleotide sequences and genomic constitution of five tryptophan genes of Lactobacillus casei. $J$ Biochem 107, 248-255

Slock J, Stahly DP, Han DY, Six EW, Crawford I P (1990) An apparent Bacillus subtilis folic acid biosynthetic operon containing $p a b$, an amphibolic trpG gene, a third gene required for synthesis of para-aminobenzoic acid, and the dihydropteroate synthase gene. J Bacteriol 172, 7211-7226

Somerville RL (1983) Tryptophan: biosynthesis, regulation, and large-scale production. In: Amino acids: biosynthesis and genetic regulation (Herrmann KM, Somerville RL, eds). Addison-Wesley Publishing Company. Reading, Massachusetts

Tschumper G, Carbon J (1980) Sequence of a yeast DNA fragment containing a chromosomal replicator and the TRP1 gene. Gene 10, 157-166

Yanofsky C, Platt T, Crawford IP, Nichols BP, Christie GE, Horowitz $\mathrm{H}$, van Cleemput M, Wu AM (1981) The complete nucleotide sequence of the tryptophan operon of Escherichia coli. Nucleic Acids Res 9, 6647-6668

Zalkin H, Yanofsky C (1982) Yeast gene TRP5: structure, function, regulation. $J$ Biol Chem 257, 1491-1500

Zalkin $\mathrm{H}$, Paluh JL, van Cleemput M, Moye WS, Yanofsky C (1984) Nucleotide sequence of Saccharomyces cerevisiae genes TRP2 and TRP3 encoding bifunctional anthranylate synthase: indole-3-glycerol phosphate synthase. J Biol Chem 259, 3985-3992 